\title{
Failure Modes of Biomedical Implants
}

\author{
Andrew Gratton, Bert Buford, Tarun Goswami, Dana GaddyKurten, and Larry Suva
}

Students: A. Buford ${ }^{1}$, A Gratton $^{1}$

Faculty Mentors : T. Goswami ', D. Gaddy-Kurten ${ }^{2}$ and L. Suva ${ }^{2}$

1. Department of Mechanical Engineering, Arkansas Tech University, Russellville, AR 72801 .

2. Center for Orthopedic Research, Department of Orthopedic Surgery, University of

Arkansas for Medical Sciences, Little Rock, AR 72205-7199.

\begin{abstract}
:
The metallic biomaterials are very well known among various biomaterials. Stainless steel was used successfully as an implant material in the surgical field, then Vitallium, cobalt based alloys were used as implant materials. Titanium is the newest metallic biomaterial among three main metallic biomaterials, stainless steels, Co-based alloys and titanium alloys. These materials are used for the instrumentation replacing failed hard tissue, for example, artificial hip joints, artificial knee joints, bone plates, dental implants and in similar applications.

Biocompatibility characteristics of titanium alloys make it the preferred choice for such applications, additional factors being light weight, excellent mechanical properties, corrosion and wear resistance. The fatigue behavior of biomedical materials is as important as other properties, yet fatigue characteristics are not considered while selecting a particular material for bio applications.

This report summarizes the fatigue behavior of biomaterials and also presents a summary from published sources where inferior fatigue resistance lead to the total joint replacement recalls. This paper reviews the fatigue, wear and corrosion resistance of biomaterials that will be useful in the design of bio prostheses.
\end{abstract}

\section{INTRODUCTION}

Mechanical failure of the human body can often be repaired by the surgical implantation of replacement synthetic parts called biological implants. An English surgeon, Sir John Charnley, is known as the inventor of the low friction artificial hip prosthesis /1/. Almost all current prostheses are variations of his original design. Current materials in complete joint replacements and degradation mechanisms in them must be explored to 
develop artificial implants that operate as well as or better than the original human component $/ 2 /$. This paper identifies one aspect of the failures of prosthesis implants: failures due to metal fatigue. Even though metal fatigue is a very specialized failure mechanism, numerous other mechanisms such as aseptic loosening, wear, fretting, corrosion, and other mechanisms may contribute to fatigue and joint failures. The types of implants that are applicable to this project are replacement hips, shoulders, spinal vertebrae, and knees.

Biological implants are generally a short-term success because biological and mechanical conflicts often cause the implants to fail. When an implant surgery is performed, there are many potential hazards that can affect the long-term outcome of the operation. The major factors that cause failure in implants are: conflicts in physical properties of the implant and the body, biocompatibility, deterioration, surgical procedures, and design failure. The primary focus of this report is to summarize biomaterials used in implants and present a summary of fatigue behavior of 1.) metals; 2.) polyethylene polymers; 3.) composites in representative environments. Due to the abundance of data, this discussion will primarily examine total hip replacement (THR) implants from a mechanical prospective. However, similar problems occur in all other forms of implants as the human body's actions produce load-time fluctuations on prostheses, causing them to cyclic/fatigue loading.

\section{TOTAL HIP REPLACEMENTS}

Each year in the United States there are an estimated 500,000 total hip replacements performed. Once a patient has gone through a THR, there is a likely chance that the patient will have problems with the implant within 5 to 15 years $/ 3 /$. These problems are most commonly caused by wear and wear particulate, migration, associated long-term loosening of the implant, fretting, and possible in vivo infections caused by the mechanical problems of the implant. The life of a THR is typically from 10 to 15 years.

An estimated $22 \%$ of revision surgeries are required on prosthesis hips in the USA alone. This translates into nearly 55000 revisions a year. It is also important to realize that $11 \%$ of the recipients are patients under the age of 40 . With the current life span of an implant being 10-15 years, this could result in up to four revision surgeries during the patient's life. An important factor in these revisions is the amount of bone area left after surgery to support the implant. A major factor that determines the amount of bone area left is the type of implant used.

There are two general categories of prosthesis hip replacements: modular and integrated. The modular implant consists of a hollow cup with a thin stem that is screwed into the cup to secure it. This process creates a small hole in the bone for attachment. Revision surgeries require an expansion of the initial hole where the primary femoral stem was fastened. This enlargement results in a loss of strength due to a reduction in bone area. If a secondary revision is required, even more problems will arise. The modular design is useful because the size of the hole required does not cause as significant of a bone area reduction as the integrated implant. 
An integrated implant has a much larger stem and, subsequently, a much larger hole in the bone. An integrated prosthesis hip is generally composed of two metallic parts: the socket, which attaches to the acetabular cup (hip bone), and the ball, neck and stem piece, which attaches to the lower femoral stem (thigh leg bone). Figure 1 shows a typical integrated THR implant.

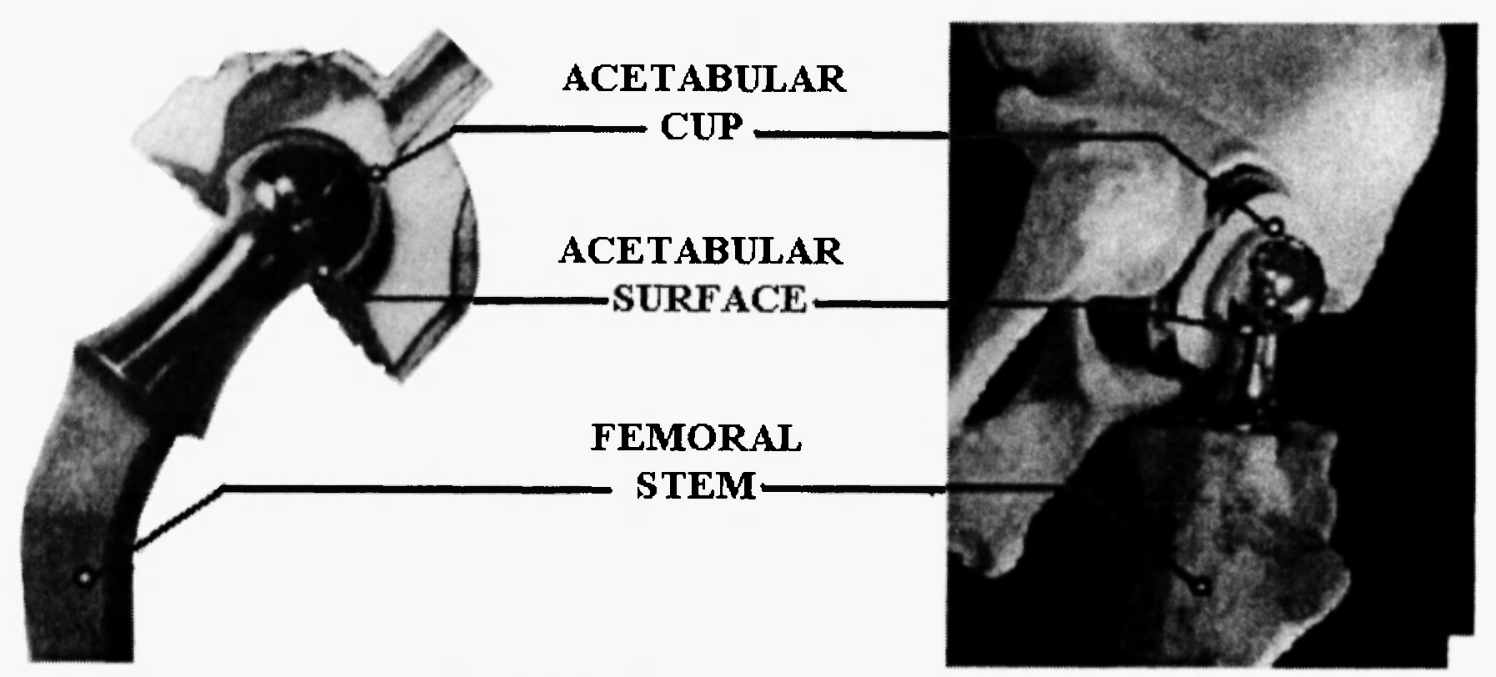

Fig. 1: Typical Integrated THR Implant /4/

Integrated implants are the predominant type of implant in use today. Much research is being conducted in the area of modular attachments and many of the material properties required by the integrated implant are also required by the modular implant, but most of the data available for research is in conjunction with integrated implants.

THR implants typically include a bearing material made of plastic (polyethylene) or other material. Either one or both portions of the prosthetic hip can be replaced. There are two methods commonly used to attach the integrated implant to the acetabular cup and the lower femoral stem. They are referred to as bone cemented fixation (cemented) and mechanical fixation (uncemented) $15 \%$. The cemented fixation holds the implant in place by cementing the prosthesis to the bone using very specialized bone cement. Mechanical fixation uses screws, bolts, nuts, plates, and wires to attach the prosthesis directly to the bone. The materials of construction and fatigue behavior of mechanical fixation accessories are not within the scope of this research and will be ignored. Another method of fixation that is typically not preferred involves the allowance of the bone to grow into the cracks and pores of the bone - a biological fixation. This method is not advisable since revision surgeries are much more difficult and painful $/ 6 \%$. A revision surgery is the surgery that is performed to either reattach or completely replace an implant that has failed.

Studies are continuously underway on the life cycle analysis of cemented and uncemented implants. The major source of data was collected by less populated European countries. These countries are ideal for studying the failure rates of implants because they have a smaller total number of replacements per capita 
than more populated countries. This allows more complete and accurate follow-ups compared to larger populations where many cases are overlooked. These countries use the predicted modes of failure in implants to gain an understanding of the implant's life expectancy. Many of the studies have essentially the same percentage conclusions when comparing the causes of failure. The surgical aspects of THRs are likely to be variables with respect to where THRs are performed, procedures used such as cemented/uncemented, topcemented etc, materials, coatings used to make THRs, and a bank of other variables due to human, engineering, and surgical factors. These issues are not discussed in this paper due to obvious reasons.

\section{NORWEGIAN STUDY}

A Norwegian study summarizes the following data for the failure rate of cemented versus uncemented replacements that required a revision surgery /7/:

Table 1

Revision Rates of Norwegian Implant Cases after 4.5 Years

\begin{tabular}{|l|c|c|}
\cline { 2 - 3 } \multicolumn{1}{c|}{} & 14009 Cemented Cases & 1326 Uncemented Cases \\
\hline $\begin{array}{l}\text { Cumulative } \\
\text { Revision Rate }\end{array}$ & $2.7 \%$ & $6.5 \%$ \\
\hline For the Cup & $0.6 \%$ & $1.7 \%$ \\
\hline $\begin{array}{l}\text { Femoral } \\
\text { Components }\end{array}$ & $1.7 \%$ & $3.9 \%$ \\
\hline
\end{tabular}

The data in the table indicates that the failure rates for the cemented cases are less than half the failure rates for the uncemented cases. This study shows the use of cemented fixation as advantageous because it has a higher success rate than the mechanical fixation. The success rate of the cemented fixation in this study was 97.3\% compared to the mechanical fixation success rate of $93.5 \% / 8 /$. It is important to understand the factors that caused the $2.7 \%$ failure rate in the cemented cases and the $6.5 \%$ failure rate in uncemented cases. This report examines the fatigue behavior of the common implant materials as a summary to use in developing a longer-lasting implant.

\section{SWEDISH REPORT}

The Swedish National Hip Arthroplasty Registry /9/ is the most detailed registry in the world, initially established as a research project in 1979. The Swedish statistics offer the most accurate long-term 
representation of hip replacement data currently available. The last biannual report was based on 169,419 primary procedures and 13,561 revisions, performed from 1979 until $1998 / 10 /$. The cemented implant is the predominant type of replacement, accounting for $93 \%$ of productivity. The revision rate is low for cemented implants and has dropped to $7 \%$, whereas it is higher for uncemented, around $13 \%$. Revision total hip replacements performed in the country were 13,561. Of those, 11,543 hips were revised for the first time and 1,713 were second-time revisions. The major reason for revision was aseptic loosening with or without osteolysis (75\% of failures). Failures caused by fatigue and fracture were nearly $5.0 \%$, implant fractures were $1.6 \%$, and polyethylene wear was $0.5 \%$. This constitutes a $7.1 \%$ failure rate attributable to fatigue and fracture. In Sweden, this percentage corresponds to about 963 revisions. Assuming that 500000 total hip replacements are performed per year in the United States, the average number of failures due a fracture and fatigue rate of $7.1 \%$ is 35500 .

The average time from for the revisions was 10 years $/ 11$. The primary modes of failure from a biological standpoint were aseptic loosening and primary deep infection. Aseptic loosening should form a significant future research endeavor as it is likely to cause wear, fretting, and therefore fatigue. The primary modes of failure from a mechanical standpoint were fracture in the bone, dislocation in the implant, fracture in the implant, and polyethylene wear. The following table summarizes the data from the report.

Table 2

Reasons for Revision

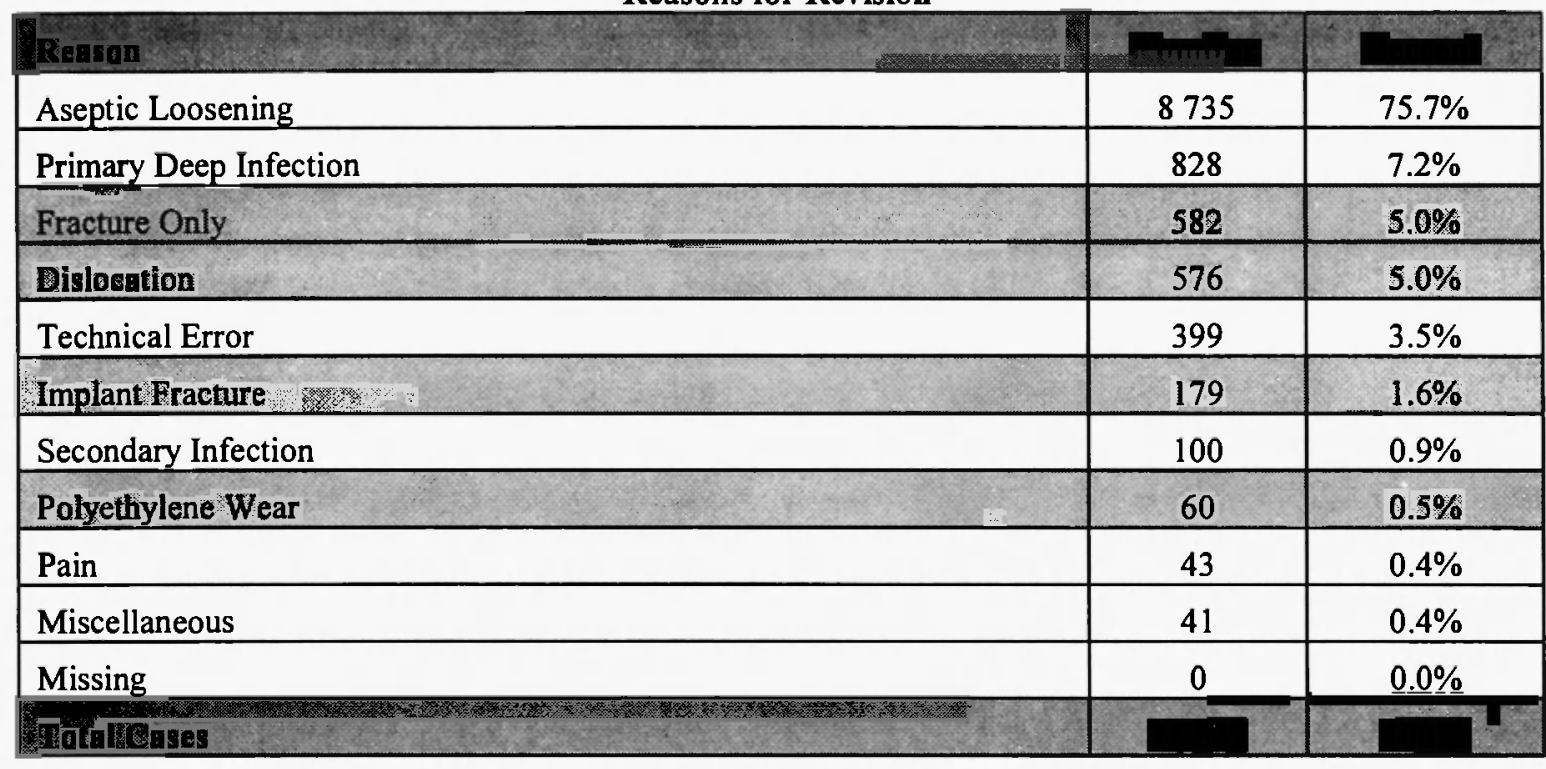

The most prevalent cause for a revision surgery in this study is fracture of the bone. This is a primary result of fatigue stress that ultimately results in failure of the bone. This failure occurs because of the reduced bone area that is lost to the implant's femoral stem. 
The report concluded that there were 179 cases of implant fracture. This was caused by yielding of the material due to stresses. Therefore it is important for implant materials to be researched to avoid failure.

\section{FAILURE MECHANISMS}

Fatigue is a phenomenon occurring under load-time fluctuations occurring at localized areas due to irreversible permanent deformation. A cyclic load is any type of varying force exerted on an object that is lessened or increased repetitively for a number of cycles. This type of failure is prevalent in prosthesis hip replacements because prostheses are subjected to cyclic stresses caused by walking, jogging, running, and other body weight movements. Figure 2 shows the devastation that can occur to an implant that is improperly designed.
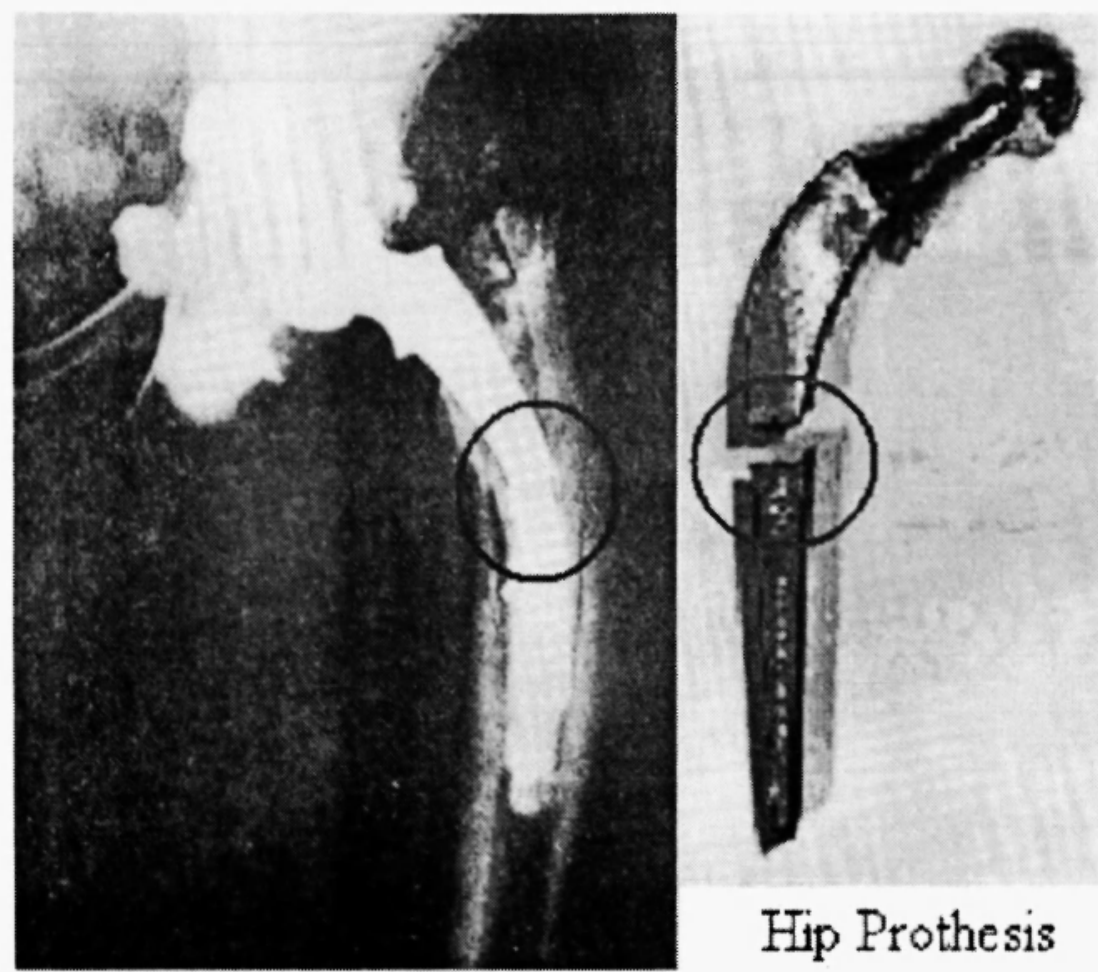

Fig. 2: Hip Prosthesis Failure

The number of cycles for an individual prosthesis depends, naturally, on the amount of activity of the individual. This often results in younger patients experiencing implant failures earlier than older patients. "For example, it is not uncommon to observe plastic deformation in bone plates possessing yield strengths of 
$40,000 \mathrm{psi"} / 12 /$. The mechanical behavior of candidate implant materials will be discussed in the following sections.

Dislocation was the next leading cause for revision in the given study. Relative motion between two surfaces constitutes bearing. The main cause of dislocation is wear of the load-bearing material in the prosthesis. The friction forces caused by grinding against the implant cause the initial formation of wear particulates. The wear particulates then act as stimuli for further formation of wear particulates. The problem causes itself to become worse. The wear particles can then cause infection, as shown in Figure 3. Wear mechanisms are also due to fretting fatigue due to the formation of a bearing. Fretting wear or fretting corrosion is the mechanism precursor to fatigue, which incubates and culminates to nucleating a "crack." These processes then transform to "fatigue."

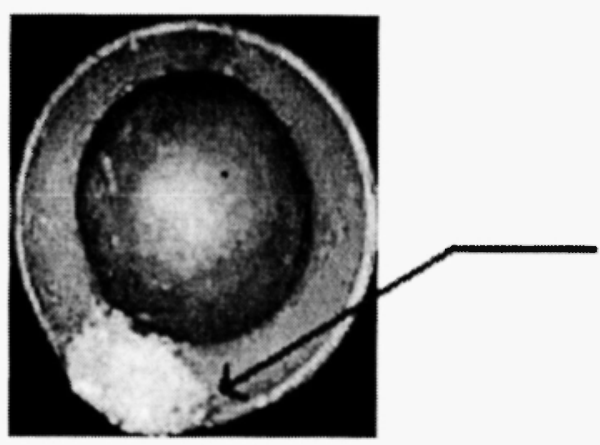

Fig. 3: Wear Particulate Infection

Polyethylene debris from an acetabular liner has been attacked by the body, resulting in giant cells that eat away bone and result in component loosening $/ 13 /$

Another problem encountered is that when the implant wears away at the bone, the bone naturally repairs itself, but not to the original shape of the bone socket considered initially for the implant. This metamorphosis of the bone then causes the problem of the implant being improperly sealed in the acetubular cup /14/. This condition ultimately can lead to the implant ball being forced outside the socket or moving the implant out of its proper location. Another complication is physical wearing of the implant. Unlike the human bone, which can biologically rebuild the outer layer of the bone that is worn, the implant cannot repair or regrow itself. Therefore any wear damage done to the implant is permanent and irreversible without a revision surgery.

Corrosion contributes to the premature failure of implants. Corrosion is a physical process by which materials return to a more stable phase, e.g. natural ore form. A material is destroyed or deteriorated by reaction with its environment normally by chemical processes. Implants are corroded by fluids in the body. The human body is approximately $80 \%$ water. "Body fluid consists of an aerated solution containing approximately $1 \%$ sodium chloride, together with minor amounts of other salts and organic compounds at 98 to $99^{\circ} \mathrm{F} " / 15 /$. This presents a problem when certain metal materials are inserted into this environment since metals generally have the tendency to corrode in the body. Therefore an implant must be able to withstand the conditions in the body without corroding significantly. Such failure cases have been reported with galvanic corrosions that release metal particles as well as fretting corrosion. Fretting corrosion is due to the 
relative motion between surfaces in corrosive environment and causes subsequent particulate formation between two dissimilar materials that results in a roughened surface forming large, deep scars $/ 16 /$.

There are fourteen independent corrosion mechanisms. Pitting, crevice, exfoliation, galvanic, stress corrosion, intergranular corrosion, and other mechanisms can develop in many different ways causing them to be of many types $/ 17 \%$. It is likely that one corrosion mechanism may interact with others giving rise to such situations as corrosion fatigue in implants. Closely related to corrosion is biological compatibility. An implant is by definition a foreign material inserted into a biological mass. The body may naturally try to reject these foreign materials; therefore, when an implant is inserted it must be compatible with the body or it generates a host of biological issues; requiring premature joint revisions.

\section{IMPLANT MATERIALS}

The alloys used in total joint components include: stainless steels, titanium alloys, cast and forged cobalt chromium molybdenum alloys, wrought cobalt chromium tungsten nickel and cobalt nickel chromium molybdenum alloys /18/. These different alloys can be grouped into three distinct categories: steel, titanium, and cobalt-based alloys. The ideal metal for a cemented component would have a high fatigue limit, yield strength, ultimate tensile strength, and corrosion resistance. Theoretically, a high modulus of elasticity may be considered advantageous because it would reduce the stress in the cement around the component and decrease the risk of cement failure, but it would be disadvantageous because the bone may become so unloaded that disuse osteoporosis, or stress shielding, could develop, resulting in cement failure and subsequent loosening of the component $/ 19 /$.

Stainless steel components were typically made of $18 \%$ chromium, $8 \%$ nickel, and $0.08 \%$ carbon content, better known as 316 steel. $316 \mathrm{~L}$ steel was developed in the 1950 s by reducing the amount of carbon content to $0.03 \%$, effectively acquiring better corrosion resistance $/ 20 \%$. Stainless steel components are only suitable for in vivo use when there is a low content of impurities and a passivated finish. Stainless steel is not readily used for implants and is inferior to other super alloys because its fatigue strength is less than other alloys, it is not as biocompatible, and is more prone to erosion $/ 21$. However, stainless steel mechanical properties have been greatly improved in recent times, making them a good alternative for elderly patients with lower expected physical demands, life expectancy, and monetary constraints.

$\mathrm{Co}-\mathrm{Cr}$ alloys are generally available with and without nickel. The Co-Ni-Cr-Mo alloy is a more recent development that is used for the stems of heavily loaded joints such as the knee and hip. Cobalt based alloys are more impervious to corrosion, fatigue wear, and fracture than iron-based alloys. The modulus of elasticity of their alloys is also higher than other materials.

Currently, titanium based alloys, especially Ti-6Al-4V and Ti-6Al-7Nb, are the most suitable material choice for prostheses and are registered in the ASTM standard as biomaterials $22,23 /$. Ti-6Al-4V is an alloy that is composed of $90 \%$ titanium, $6 \%$ aluminum, and $4 \%$ vanadium. This alloy is a superior material due to 
its corrosion resistance, high endurance limit, high strength to weight ratio, and positive biocompatibility properties. However, titanium has poor shear strength, making it less desirable for bone screws, plates, and similar applications. Titanium also tends to seize when in sliding contact with itself or other metals $/ 22 /$. Also, titanium has a high coefficient of friction, so it is often not used for two components in direct contact with one another since wear particles are readily formed. Titanium is very corrosion resistant due to a solid oxide layer (the only stable reaction product) formed under in vivo conditions $/ 23 /$. Ti-6Al-7Nb contains $7 \%$ niobium instead of vanadium. This alloy has better corrosion resistance than Ti-6Al-4V.

There are many alloys of titanium available for use, each with individual characteristics that make it a good solution to a certain problem. However, by making one characteristic better, other characteristics may become weaker. Figure 4 shows how the modulus of elasticity varies for different titanium alloys.

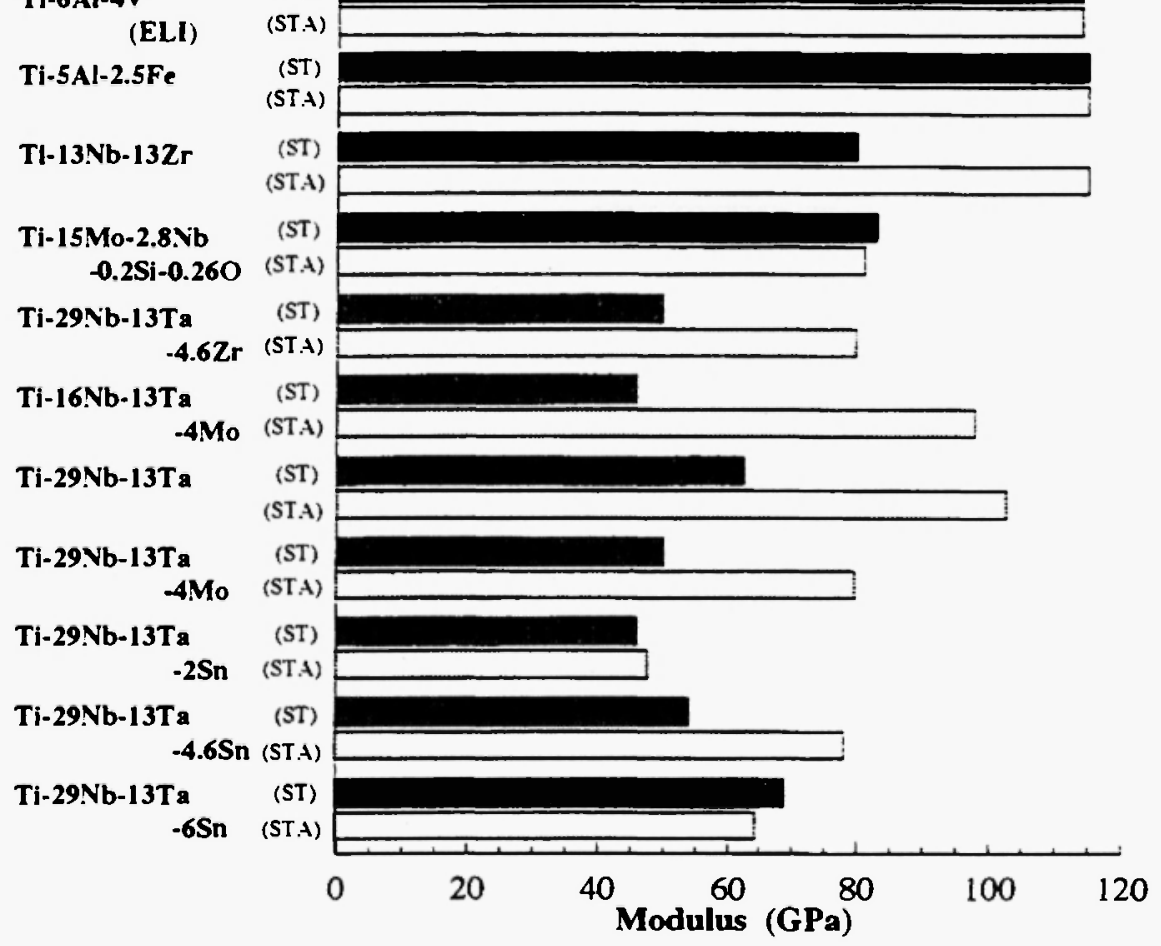

Fig. 4: Modulus of Elasticity of Varying Titanium Alloys /24/.

Figure 5 shows how the tensile strength of the different titanium alloys also varies. 


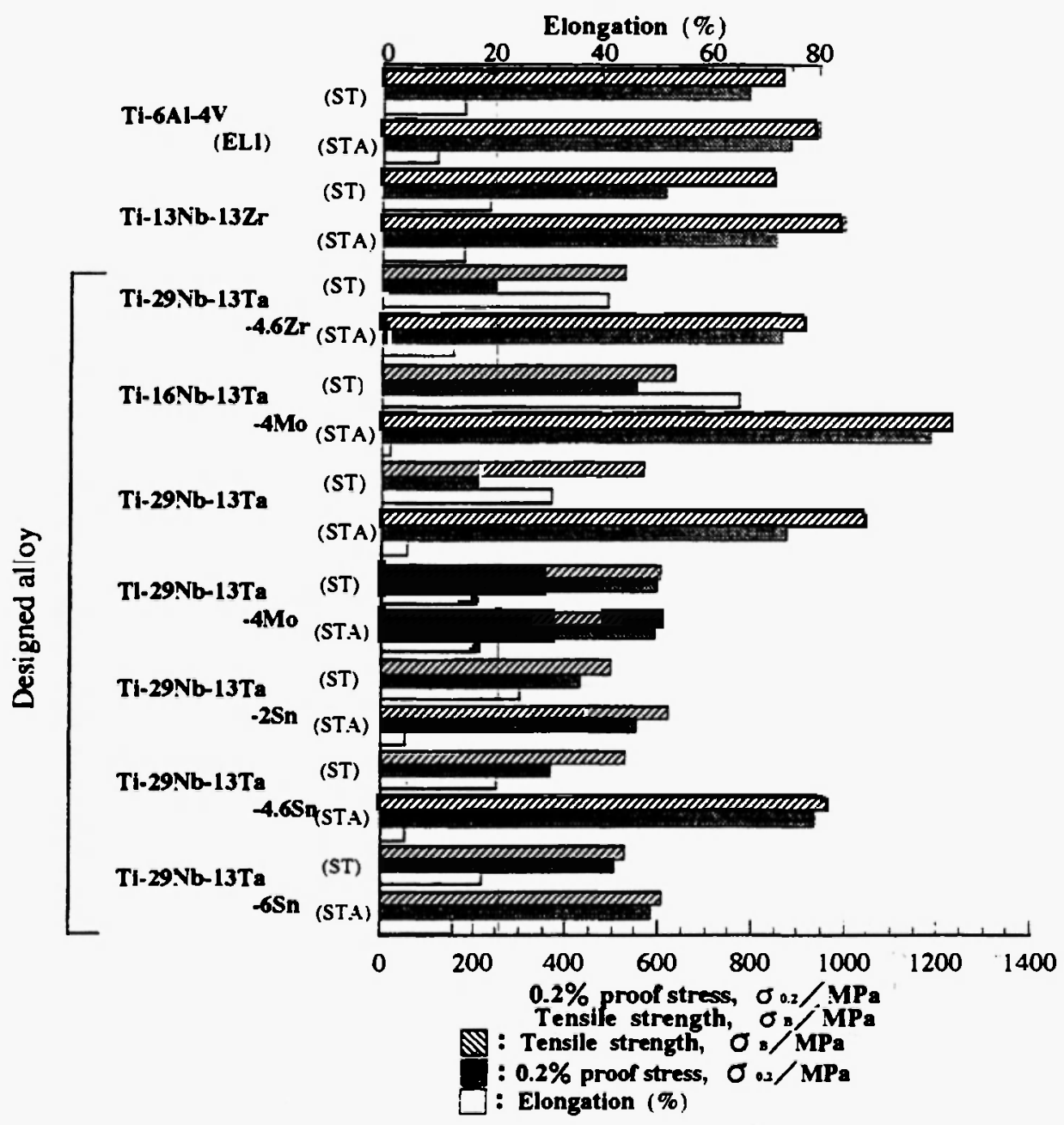

Fig. 5: Tensile Strength of Varving Titanium Allovs /25/

Although titanium alloys seem ideal as implant materials, there has not been a significant amount of longterm analyses performed, since these materials have only recently been introduced for prostheses. The use of titanium alloys in other applications has shown significant improvement over steel alloys; therefore, there is a promising outlook for the material. Since most failures occur only after a few years, it is still too soon to determine the material's exact successfulness.

A prosthesis made with titanium alloys is susceptible to many damage mechanisms. The forces arising from body weight and movement may give rise to a force which may produce stresses in the cup and stem in integrated areas such as head-neck joints and may cause displacements in modular head-neck prostheses. This relative motion even in the order of a few micrometers may produce fretting and vibration of the debris. 
This debris, even in very small concentrations (ppm), may aid medical conditions that are of a biological nature requiring revision. Therefore, both modular and integrated prostheses are currently being studied for possible application in THR's.

Another aspect of implant material properties is the damage tolerance characteristics of titanium alloys. Given that other damage criteria may not evolve into a damage mechanism, fatigue studies for both S-N fatigue behavior and crack growth studies for titanium are very important. Data from present work provides a summary of stress amplitude required to cause failure at a particular number of life cycles. It must be pointed out that the S-N fatigue behavior of a material degrades as the environment changes from a vacuum to standard air pressure, as humidity levels increase, and as corrosive elements in the environment react with the metal under study. Goswami /26/ published the fatigue crack growth behaviors in Ti-6Al-4V. The data is presented below for higher humidity levels at room temperature.

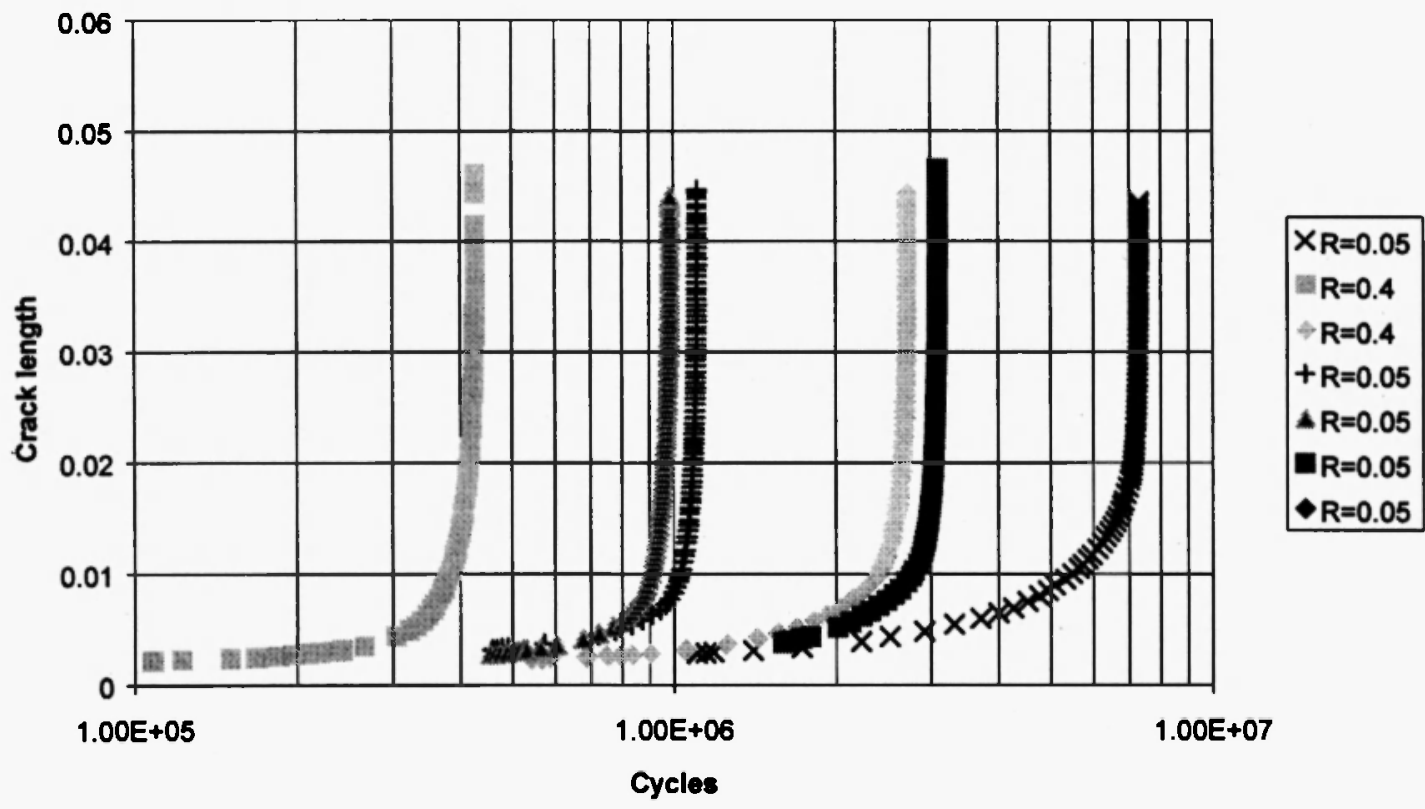

Fig. 6 : Crack length versus number of cycles for Ti- $6 \mathrm{Al}-4 \mathrm{~V}$ high humidity tests.

From Figure 6, the crack lengths of $\mathrm{Ti}-6 \mathrm{Al}-4 \mathrm{~V}$ become longer as the number of cycles on the specimen increases. At a certain number of cycles, the crack spontaneously increases at alarming rates. The R-value indicates the stress ratio of minimum stress to maximum stress. An R-value of less than 0.5 is a conservative estimation of the stress ratios in prostheses. The figure shows data for a high humidity test environment. The data is applicable to biomaterials because in vivo conditions are best modeled as high humidity. This significant increase in crack length propagation applies to the material's ultimate failure due to cracking, a severe result of fatigue wear. 
The rate of crack growth rate can also be studied. Figure 7 illustrates the rate of crack growth for Ti-6Al$4 \mathrm{~V}$.

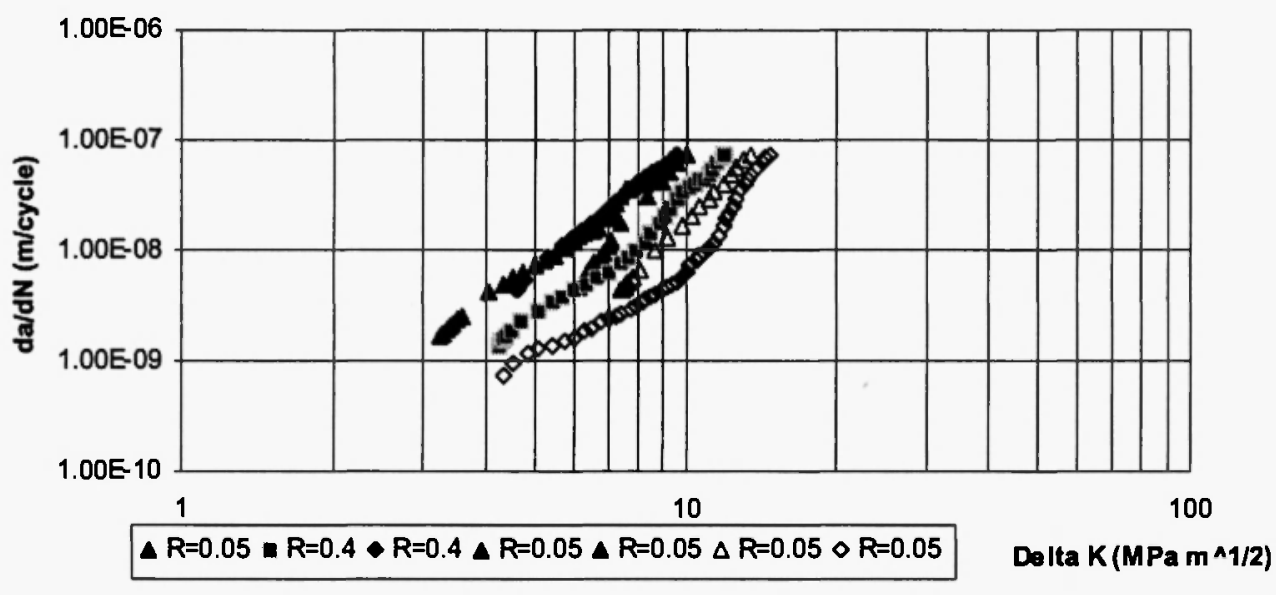

Fig. 7 : Crack propagation behavior of Ti-6Al-4V in high humidity environment

The data in Figure 7 indicates that the rate of crack growth increases as Delta $\mathrm{K}$ increases.

The following table shows a list of current metallic biomaterials suitable for implants and a comparison of their mechanical behavior.

\begin{tabular}{|c|c|c|c|c|c|c|c|c|}
\hline Material & $\begin{array}{c}\text { Yield Strength } \\
(\mathrm{ksi})\end{array}$ & $\begin{array}{c}\text { Temperature } \\
\text { Range }\left({ }^{\circ} \mathrm{F}\right)\end{array}$ & $\begin{array}{c}\text { Maximum } \\
\text { Toughness }\end{array}$ & $\mathrm{F}_{\mathrm{tu}}(\mathrm{ksi})$ & $\mathrm{F}_{\mathrm{ry}}(\mathrm{ksi})$ & $\mathrm{F}_{\mathrm{cy}}(\mathrm{ksi})$ & $\mathrm{F}_{\mathrm{su}}(\mathrm{ksi})$ & $\mathrm{F}_{\mathrm{bru}}(\mathrm{ksi})$ \\
\hline Ti-6Al-4V & $121-145$ & -1070 & Annealed & $130-160$ & $120-145$ & $124-162$ & $79-100$ & $206-308$ \\
\hline Ti-6Al-7Nb & - & - & - & - & - & - & - & - \\
\hline L-605 & - & - & - & $125-135$ & $45-62$ & $41-61$ & $88-95$ & $186-241$ \\
\hline SS 316 & - & - & - & - & - & - & - & - \\
\hline SS 316L & - & - & - & - & - & - & - & - \\
\hline SS 304 & - & - & - & - & - & - & - & - \\
\hline & & & & & & & & \\
\hline Material & $\mathrm{F}_{\mathrm{bry}}(\mathrm{ksi})$ & $e$ & $\left(10^{3} \mathrm{ksi}\right)$ & $\mathrm{ksi})$ & $\mathrm{ksi})$ & $\mu$ & $\omega$ \\
\hline Ti-6Al-4V & $164-243$ & $5-10$ & 16.0 & 16.4 & 6.2 & 0.31 & 0.16 & $650 \mathrm{MPa}$ \\
\hline Ti-6Al-7Nb & - & - & - & - & - & - & - & $700 \mathrm{MPa}$ \\
\hline L-605 & $88-123$ & $30-45$ & 32.6 & 32.6 & 12.6 & 0.29 & 0.33 & - \\
\hline SS 316 & - & - & 28 & - & - & - & 0.288 & \\
\hline SS 316L & - & - & - & - & - & - & - & - \\
\hline SS 304 & - & - & - & - & - & - & - & - \\
\hline
\end{tabular}


Much of the data in the table was obtained from the MIL handbook, the military standard specification for materials. Unfortunately, the handbook does not provide information for all of the materials under consideration for implants. In fact, many of the materials listed do not have any significant amount of research material available. For this reason, many of the materials do not have complete data sets. The table will be completed as more research is performed.

For fatigue strength, the best material is a wrought cobalt chrome alloy while the purest grade of commercially available pure titanium shows the lowest values /27/. Premature fatigue wear is generally caused by poor workmanship and handling or a poor design. During surgery and in general handling, scratches will inevitably appear on the surface of the prosthesis. These scratches result in an intensification of the stress at those points; this phenomenon is called notch sensitivity. The stresses collect at the imperfections and provide a location for crack growth propagation. It seems the best hope to overcome fatigue wear and eventual crack growth is in the development of fiber-reinforced materials, which show remarkable resistance to fatigue $/ 28 /$.

\section{DESIGN CRITERIA}

Prosthesis design consists of 3 design parameters: fatigue and fracture, corrosion, and wear. A prosthesis should be able to withstand 3 million cycles per year for the average sedentary individual. Fatigue analysis must take into consideration other factors that will prematurely weaken the material, such as corrosion. In addition, combined effects of material failure modes working together must be considered as well. A modern method of estimating the maximum stresses and fatigue life of a prosthesis is through the finite element method. This method splits an object into a finite number of elements that can then be subjected to a load. The stresses are then calculated using the finite element method. Also, changes in design can be easily made and the stresses found in a relatively short amount of time. An example of a finite element analysis of a hipbone is shown in Figure 8.

Abicus and Algor are packages used for joint analysis at the Orthopaedic Research Laboratory at the Scripps Clinic in San Diego. They have the advantage of analysis with non-linear materials, suitable for the changes in material properties across the cross-section of cancellous and cortical bone. Within most packages, the bone properties must be assumed to be linear elastic, isotropic, and homogeneous. Cancellous bone is actually extremely anisotropic and inhomogeneous. Cortical bone, on the other hand, is approximately linear elastic, transversely isotropic and relatively homogenous. To account for these properties, different values need to be assigned to each individual element $/ 30 \%$. 
Pelvis-Standing 4Ca N : Procest-fiends Nixdrat Sress Unthe: Nan'2

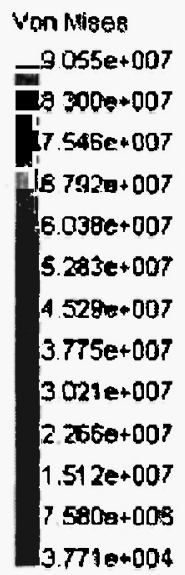

Fig. 8: Sample finite element analysis of a hip bone with $400 \mathrm{~N}$ force applied $/ 29 /$.

Unfortunately, the finite element analyses performed do not represent exact load conditions of the human body. Non-linear elements such as bone density or the directional differences in Poisson's ratio for bone cannot be modeled into the analysis. Also, the exact shape of parts is impossible to reproduce in a computer program since the size and shape of the human skeletal system is continuously growing and changing. For instance, an individual who decides to lift weights when his previous primary physical activity was running, will experience a reorganization of his skeletal system to accommodate the change in load type and distribution. As technology advances, however, the computer-generated models become more accurate and will eventually represent a very good modeling and design tool to develop better prostheses.

A major concern with all joint replacements is the degradation of the metals used $/ 31 /$. Corrosion is the undesirable chemical reaction of the metal with its harsh environment. Corrosion products can cause accelerated formation of wear fragments, which can lead to loosening of the components or other problems related to the spread of toxic elements in the body. A recent study has demonstrated an eight fold increase in chromium levels in serum from patients with long term metal on metal THRs compared with control groups 132 . In addition to trace element findings, chances of related biological problems are also increased. "A 1970's study discovered that dialysis patients from certain parts of the country accumulated aluminum derived from tap water in the cytoplasm of brain cells. The primary effect of this accumulation was damage to the brain, which manifested itself as speech disturbances, myochronic jerks, motor apraxia, memory 
disturbances, personality changes, dementia, and seizure disorders (dialysis encephalopathy). The secondary effects of high levels of aluminum in dialysis patients is the development of osteomalacia where aluminum displaces the calcium in the osteoid resulting in an increased calcium level in the blood stream leading to an inhibition of parathyroid hormone" 133 . The most common titanium alloy used for prosthesis implants contains $6 \%$ aluminum. Because of the number of physical and mechanical problems caused by corrosion and corrosion products, it is necessary to design prostheses with very corrosion-resistant materials. Non-toxic elements that will become more prevalent in implant design will be: $\mathrm{Ti}, \mathrm{Nb}, \mathrm{Ta}, \mathrm{Zr}$ and $\mathrm{Sn}$, which are low cytotoxic elements $134 /$.

\section{CONCLUSION}

The information provided in this review shows that mechanical and material issues are very important in the design and selection of materials to prepare bio-prostheses. Failure of implants is due to biochemical and mechanical reasons. It is arguable that mechanical factors may contribute to the development of such problems as aseptic loosening or vice-versa due to dislocation of the mechanical axis of the implant and wear between metal-to-metal or polyethylene-to-metal contacts that may also generate fretting. Materials issues in the design and development of new modular and integrated implants are very important and this paper attempts to summarize those issues. Fatigue should become one of the major design criteria together with biological (biocompatibility) factors in the design of implants. The impact of additive failure modes is a difficult area of material research. Knowledge of these factors will help design better implants for longer life and durability.

\section{RECOMMENDATIONS}

The following recommendations are made for continuation of this research.

1. Polyethylene wear in the presence of chrome cobalt, titanium, steel, and stainless steel should be reviewed/examined.

2. The fatigue behavior of biomedical materials needs to be summarized. The data will have a direct link to the life expectancy of the implant.

3. The relationship between aseptic loosening and the mechanical behavior of the joint materials needs to be defined.

4. The effects of the controlled environment on materials have not been cited in the literature. Further work on wear, fretting, and fatigue behavior is recommended.

5. Biomaterials and their mechanical behavior such as tensile properties, fatigue, wear, fretting etc. need more research. 
6. Finite element modeling and analysis of relative displacements of implants will be conducted in the future so that presence of wear and fretting mechanisms be identified from the early stages of implant design and/or that are in vivo applications. This may help identify those implants that may need revisions.

\section{REFERENCES}

1. Piehler, H.R. "The Future of Medicine: Biomaterials." http://www.mrs.org/publications/bulletin/2000/aug/Piehler.pdf, Web Page 2000

2. Petersen, S. "The Design and Development of Hip Replacements." http://www.geocities.com/hip_replacements, Web Page 2001

3. Private discussion with surgeons and implant manufacturers.

4. Ibid [2]

5. Park, J.B. "Hip Joint Prosthesis Fixation-Problems and Possible Solutions." The Biomedical Engineering Handbook: Second Edition, Ed. Joseph D. Bronzino, Boca Raton: CRC Press LLC, 2000, 46.1-46.3

6. Private discussion with surgeons from University of Arkansas for Medical Sciences, Center for Orthopaedic Research.

7. Ibid. [1]

8. Norwegian National Hip Arthroplasty Registry (http://www.haukeland.no/nrl/).

9. Malchau H, Herberts P, Soderman P, Oden A "Prognosis of Total Hip Replacement", Department of Orthopedics Goteborg University, Sweden, The Swedish National Hip Arthroplasty Registry, 2000, Stats Ref. For Swedish Report

10. Ibid. [9], page 3 .

11. Ibid. [9], page 3.

12. Fontana, M.G. "Corrosion Engineering", Chapter 8-11, Other Environments, Human Body., Copyright 1967 McGraw-Hill Inc, p. 288

13. Ibid. [1]

14. Baron, Roland, D.D.S. Ph.D. "Primer on the Metabolic Bone Diseases and Disorders of Mineral Metabolism", American Society for Bone and Mineral Research: Fourth Edition, Ed. Murray J. Favus, M.D., Lippincott Williams \& Wilkins Chapter 1

15. Ibid. [11], page 288 .

16. Ibid. [11]

17. Hendry JA, Pilliar RM "The fretting corrosion resistance of PVD surface-modified orthopedic implant alloys." Institute of Biomaterials and Biomedical Engineering. The University of Toronto, 170 College St. W., Toronto, ON M5S 3E3, Canada. J Biomed Mater Res 2001; 58(2): 156-66 
18. Sweetnam, D. "Metals in Orthopedic Surgery." Institute of Orthopaedics, London. Brockley Hill, Middlesex, HA7 4LP. (http:// www.fractures.com/institute/bme/design.htm)

19. Ibid. [18]

20. Ibid. [18]

21. Ibid. [18]

22. ASTM, Designation F136-82. "Standard Specification for Wrought Titanium 6 Al 4V ELI Alloy for Surgical Implants." ASTM, Philadelphia, PA, 1994, 19-20.

23. ASTM, Designation F136-82. "Standard Specification for Wrought Titanium 6 A 7Nb ELI Alloy for Surgical Implants." ASTM, Philadelphia, PA, 1994, 687-689.

24. Ibid. [18]

25. Ibid. [18]

26. Goswami, T. "Crack Growth Behavior of Titanium Alloys." Mechanical Engineering Department, Arkansas Tech University, Russellville, AR 72801

27. Kuroda D., Niinomi M., Morinaga M., Kato Y., Yashiro T. "Design and mechanical properties of new $\beta$ type titanium alloys for implant materials." Materials Science and Engineering A243 (1998), 244-249. Elsevier Science S.A.

28. Ibid. [27], pages 247-249.

29. Petersen, S. "The Design and Development of Hip Replacements." http://www.geocities.com/hip_replacements/fea_results.htm Web Page 2001

30. Petersen, S. "The Design and Development of Hip Replacements." http://www.geocities.com/hip_replacements Web Page 2001

31. Black J. "Does Corrosion Matter." J Bone and Joint Surg. (Br). 1988;70 (B): 517-520.

32. Jacobs J.J., Skipor A.K., Doorn P.F., Amstutz H.C. $42^{\text {nd }}$ Annual Meeting, Orthopaedic Research Society. 1996;21:498.

33. Ibid. [18]

34. Y. Okazaki, Y.Ito, K.Kyo, T.Tateisi, Mater. Sci. Eng. A213 (1996 138-147). 
